\title{
Can in-hospital or post discharge caregiver involvement increase functional performance of older patients? A systematic review
}

Margaretha van Dijk ${ }^{1 *}$ (D, Jasmien Vreven ${ }^{1}$, Mieke Deschodt ${ }^{2,3,4}$, Geert Verheyden ${ }^{5}$, Jos Tournoy ${ }^{2,6}$ and Johan Flamaing 2,6

\begin{abstract}
Background: Regaining pre-hospitalization activity levels is only achieved in 30-50\% of older patients. Extra physiotherapy time has been proven to improve functional outcome and shorten length of stay, but is costly. Considering their key role in caring for older people, involving informal caregivers in rehabilitation might further improve functional performance.

Aim: To determine if in-hospital or post discharge caregiver involvement can increase functional performance in older adults. The secondary aim was to determine if caregiver involvement can influence, quality of life of patient and caregiver, medical costs, readmission rate, discharge location, and mortality.

Design: Systematic review with narrative synthesis.

Methods: The electronic bibliographic databases MEDLINE, Embase, CINAHL, Cochrane and Web of Science were searched for (quasi) experimental and observational studies, with the following inclusion criteria; caregiver involvement regarding functional performance, mean age over 65 years, admitted to a hospital unit and subsequently discharged to their home setting. Risk of bias was assessed with the Rob 2 (randomized trials) and the ROBINS-1 tool (non-randomized studies).

Results: Eight studies of an initial 4683 were included: four randomized controlled trials, one prospective cohort study, one non-randomized controlled trial, one subgroup analysis of an RCT and one prospective pre-post study. All but one study included patients with stroke. Three types of caregiver interventions could be distinguished: a care pathway (inclusion of caregivers in the process of care), education on stroke and teaching of bed-side handling-skills, and caregiver-mediated exercises. The one study evaluating the care pathway reported $24.9 \%$ more returns home in the intervention group. Studies evaluating the effect of education and bed-side handling-skills reported higher effect sizes for several outcomes with increasing session frequency. All studies with caregiver-mediated exercises showed beneficial effects on functional performance, immediately after the intervention and within 3 months follow-up.
\end{abstract}

Conclusion: The findings of this review suggest that involvement of caregivers in the rehabilitation of older adults leads to better functional performance up to 3 months after initiation. However, evidence is low and mainly focusing on stroke.

Keywords: Caregiver involvement, Older adults, Functional performance, Hospitalization, Physiotherapy

\footnotetext{
* Correspondence: Margaretha.vandijk@uzleuven.be

'Department of Physical Medicine and Rehabilitation, UZ Leuven - University

Hospitals Leuven, campus Pellenberg, Weligerveld 1, 3212 Pellenberg,

Belgium

Full list of author information is available at the end of the article
}

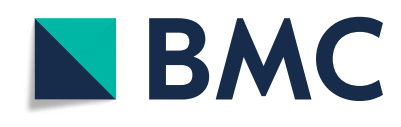

(c) The Author(s). 2020 Open Access This article is licensed under a Creative Commons Attribution 4.0 International License, which permits use, sharing, adaptation, distribution and reproduction in any medium or format, as long as you give appropriate credit to the original author(s) and the source, provide a link to the Creative Commons licence, and indicate if changes were made. The images or other third party material in this article are included in the article's Creative Commons licence, unless indicated otherwise in a credit line to the material. If material is not included in the article's Creative Commons licence and your intended use is not permitted by statutory regulation or exceeds the permitted use, you will need to obtain permission directly from the copyright holder. To view a copy of this licence, visit http://creativecommons.org/licenses/by/4.0/. The Creative Commons Public Domain Dedication waiver (http://creativecommons.org/publicdomain/zero/1.0/) applies to the data made available in this article, unless otherwise stated in a credit line to the data. 


\section{Background}

Functional decline including reduced abilities of selfcare are common consequences of hospitalization among older adults [1]. Up to $39 \%$ of patients 75 years or older admitted to geriatric, medical and surgical wards experience functional decline [2, 3]. The frequency of functional decline increases with age and is associated with the failure to recover to the basic activities of daily living (ADL) level during hospitalization [3]. Inactivity, immobility, suboptimal continence care, and poor nutrition during hospitalization have been suggested as the main reasons for functional decline $[1,4]$.

Physical therapy (PT) has proven to be effective in improving functional performance in older hospitalized patients [5]. A systematic review by Kosse et al. indicated that early physical therapy and rehabilitation can be safely executed and leads to better performance of ADL, shorter length of stay, and higher proportion of discharge home in hospitalized older adults [6]. Geriatric rehabilitation is best seen as a holistic treatment with a functional and multidisciplinary approach as well as long term health promotion [7-9]. Since increased physical activity is associated with arresting or reversing declines in functional performance, patients should be guided to seek opportunities and use available tools to achieve an active lifestyle $[8,9]$.

New hospital acquired ADL disability, is associated with the inability to regain pre-hospital functioning [10-12]. Only between 30 to $50 \%$ of the older patients regain prehospitalization activity levels and $25 \%$ of older adults treated in an inpatient rehabilitation facility are readmitted soon after discharge [13]. Limited time of PT, lack of patient activity outside therapy and detraining (deterioration of the acquired level of performance) when therapy has stopped are all reasons for functional decline, both during hospitalization and after discharge [14-17]. Extra PT time improves functional outcome and shortens length of stay, but consequently increases healthcare costs [18]. Even when PT meets the WHO activity guideline of $300 \mathrm{~min}$ of activity at moderate intensity per week, it still is insufficient to change sedentary behavior $[19,20]$. At the same time, it is often difficult for hospitalized older patients to be active on their own, due to, among others, their dependency of healthcare workers (HCW) for transfers and walking. In addition, patients are discharged as soon as a basic level of functional performance is met, like getting in and out of bed [21]. Consequently, at the moment of discharge the patient's functional performance level is lower than their pre-hospital level. This in turn, makes patients and caregivers cautious about taking up normal activities or even continuing exercises.

Informal caregivers play an important role in care of older people in Europe as no care system will ever be able to completely cover all long-term care needs by professional health care services [22]. A meta-analysis by Sörensen et al. shows that different interventions with caregivers are effective in lowering caregiver burden, depression, care receiver symptoms, and in improving subjective well-being, perceived caregiver satisfaction and ability/knowledge [23]. Although several studies evaluated the effect of caregiver involvement on functional performance in older adults, no systematic review on this subject has been undertaken.

The aims of this systematic review are:

1) To determine if in-hospital or post discharge caregiver involvement can increase functional performance in older adults.

2) To determine if caregiver involvement regarding functional performance can influence quality of life of patient and caregiver, need for professional help, readmission rate, discharge location, healthcare utilization and mortality.

\section{Methods}

The review protocol was registered in PROSPERO (CRD42018091798) and the PRISMA reporting guidelines were used. (www.prisma-statement.org).

\section{Search strategy}

Multiple search strategies were used for this review. The electronic bibliographic databases MEDLINE using PubMed, Embase, CINAHL, Cochrane and Web of Science were searched using a comprehensive search string on the fourth of December 2018 (Additional file 1). We limited our search to studies reporting in English, Dutch, German and French. There was no limitation with regard to the date of publication. In addition, the reference lists of relevant papers and related reviews were screened. Case reports, letters and editorials were manually excluded from the search results. On the sixth of April 2020 PubMed was searched again to identify potential new studies.

\section{Selection of relevant papers}

All studies reporting on caregiver involvement regarding functional and physical performance activities in (recently) hospitalized older adults were of interest. Studies were included if they studied a sample of older adults aged 65 years or older (or with a mean age over 65 years old) admitted to a hospital unit and subsequently discharged to their home setting. Caregivers were defined as unpaid members of a person's social network helping the patient with ADL. Studies using a randomized controlled design or a quasi-experimental design (including non-randomized controlled studies, before-and-after studies retrospective and prospective, and interrupted 
time series) with follow-up to twelve months were included.

Studies in nursing home residents and community dwelling older adults without a recent hospitalization, and reports on interventions with professional healthcare workers, were excluded. As standard of care can be expected to differ in light of international differences, no a-priori exclusion criteria were formulated in relation to the comparison group.

Two reviewers (JV, MV) screened the search results independently, using Endnote. First, titles and abstracts were screened, using the predetermined in- and exclusion criteria. If the titles and abstracts did not provide enough information the full text was read and screened independently by two reviewers to determine eligibility. In case of disagreement of study eligibility, the two reviewers discussed their findings and a third reviewer (MD) was consulted to reach an agreement.

\section{Data extraction and synthesis}

One reviewer (JV) extracted data from the included studies and a second reviewer (MV) checked the completeness and correctness of the extracted data. The following data were extracted: first author, year of publication, country and setting, study design, study population, detailed description of the intervention (i.e. education, exercises, number of hours and/or sessions), outcome measures and definitions, time measurements, and follow-up after the end of intervention (Table 1). The results of the individual studies were tabulated and grouped according to four different outcomes (Tables 2 and 3 and Additional files 3 and 4). Results are summarized by means of a narrative synthesis.

Risk of bias of randomized controlled trials (RCTs) and cluster RCTs was evaluated by one reviewer (MD) at the study level with the revised Cochrane risk of bias tool (Rob 2) which grades the risk of selection, performance, attrition, detection and reporting bias, while risk of bias of the non-randomized studies was evaluated with the ROBINS-I tool $[24,25]$.

\section{Results}

\section{Article selection}

A total of 4683 studies were screened for eligibility (Fig. 1). After excluding 4669 articles based on title and abstract, 15 full-texts were read to determine eligibility. A total of nine articles reporting on eight studies were analyzed [26-34]. Two articles reported on the same study and subjects and they will be referred to as one study $[32,33]$. Additionally, two protocol papers of included studies were available, which we used for data on characteristics and risk of bias [35, 36]. Another 144 studies were screened for eligibility after the search on the sixth of April 2020, but none was withheld.

\section{Risk of bias}

The four included RCTs did not show a high risk of any form of bias on any domain, but there were concerns regarding performance and detection bias [27, 28, 32-34]. The non-randomized studies all showed a high risk of bias due to confounding [26, 29-31]. Two of the nonrandomized studies also scored high for selection bias $[30,31]$ and two scored high on bias due to missing data [26, 31]. Additional file 2 shows all the results of risk of bias assessment.

\section{Characteristics of the included studies}

We included four RCTs [27, 28, 32-34], one prospective cohort study [26], one non-randomized controlled trial [29], one subgroup analysis of an RCT [30] and one prospective pre-post study [31]. Six of the studies were performed in Europe [26-29, 31-33], one in Canada [30] and one in Australia [34]. Seven studies included only patients with stroke [27-34] and one included geriatric patients with complex health problems [26]. Sample sizes varied from 40 to 928 patient-caregiver dyads. One study had no follow-up (last measurement was at the end of the intervention) [30] and three studies had 12month follow-up [27, 31-33]. In two studies the first outcome measure point, which was called baseline, was at discharge, after the intervention [29,31]. The study of Forster et al. evaluated the same intervention as Kalra \& Patel et al., but in another setting and other study sample [27, 32, 33]. More information regarding the included studies can be found in Table 1 .

\section{Reported outcome measures}

All studies measured functional performance, which in turn can be subdivided into basic functional performance (ADL) and extended functional performance. Six studies also used a variety of other outcomes (caregiver burden, quality of life, patient and/or caregiver depression, length of stay and initial hospitalization costs), which can be grouped into psychological well-being and others.

\section{Basic functional performance}

Basic functional performance was most often measured by the Barthel Index (BI) [28, 29, 31-34] and the Nottingham Extended ADL [27, 28, 31, 34]. Other outcome measures used were the Frenchay Activities Index (FAI) [26, 32, 33], the Katz index (KI) [26], the Reintegration to Normal Living Index (RNLI) [28] and the Modified Rankin Scale (MRS) [31]. An overview can be found in Table 2.

\section{Extended functional performance}

Extended functional performance outcome measures were quite diverse and nearly every study used different ones. Lower limb function was measured with the Lower Limb of Fugl Meyer Assessment (LL-FMA) [28, 34], gait 
Table 1 Characteristics

\begin{tabular}{|c|c|c|c|}
\hline $\begin{array}{l}\text { Author } \\
\text { Year } \\
\text { Country }\end{array}$ & $\begin{array}{l}\text { Design (D) } \\
\text { Setting (S) } \\
\text { Sample size (SZ) }\end{array}$ & Target patient population & $\begin{array}{l}\text { Intervention (I) } \\
\text { Duration (D) } \\
\text { Frequency (F) }\end{array}$ \\
\hline $\begin{array}{l}\text { Everink } \\
\text { et al. } \\
2018 \\
\text { The } \\
\text { Netherlands }\end{array}$ & $\begin{array}{l}\text { D: Prospective } \\
\text { cohort study } \\
\text { S: Hospital, } \\
\text { geriatric } \\
\text { rehabilitation } \\
\text { facility, community } \\
\text { SZ: } 149 \text { patients } 54 \\
\text { caregivers }\end{array}$ & $\begin{array}{l}\text { Geriatric patients (> } 65 \text { years and } \\
\text { complex health problems)Admitted } \\
\text { to a geriatric rehabilitation } \\
\text { facilityCommunity-dwelling prior to } \\
\text { hospital admission }\end{array}$ & $\begin{array}{l}\text { I: Integrated Care Pathway: Process } \\
\text { of care during the trajectory of } \\
\text { hospital admission, discharge to } \\
\text { geriatric rehabilitation and discharge } \\
\text { back to community. Patients and } \\
\text { their caregiver are actively involved } \\
\text { in the triage decision. } \\
\text { D: Period of hospitalization (acute } \\
\text { and rehabilitation) until discharge. F: } \\
\text { Not stated }\end{array}$ \\
\hline
\end{tabular}

$\begin{array}{ll}\text { Forster } & \text { D: Multicentre } \\ \text { et al. } & \text { cluster RCT } \\ 2013 & \text { S: } 36 \text { stroke units } \\ \text { UK } & \text { in four } \\ & \text { geographical } \\ & \text { regions. } \\ & \text { SZ: } 928 \\ & \text { patients } 928 \\ & \text { caregivers }\end{array}$

Galvin et al. D: RCT

2011 S: 6 acute

Ireland hospitals

SZ: 40 patients 40

caregivers

\author{
Gräsel et al. D: Non- \\ $\begin{array}{ll}2005 & \text { randomized con- } \\ \text { Germany } & \text { trolled trialS: } 2\end{array}$ \\ study wards of a \\ rehabilitation clinic \\ SZ: 71 patients 71 \\ caregivers
}

Patient with stroke Medically stable I: London Stroke Carers Training Likely to return home

Patient with strokeNo cognitive impairment Participate in a physiotherapy program in knowledge or skills essential for Intervention manual and caregiver training record.

D: Period of hospitalization F: Not stated friend with the skills necessary to
Patient with strokeFunctional deficit Required treatment in rehabilitation clinic
Patient with strokeActive scapular elevationFM scale 10-57
2010

Canada
SZ: 50 patients 50

caregivers
Hebel et al. D: Prospective pre- Patient with stroke

2014

Poland post study

S: Hospital

SZ: 243 patients

243 caregivers

\section{Course:Assessment of competencies} day-to-day management of disabled survivors of stroke (14 components).

I: Family-Mediated Exercise Intervention:Training the family member/ carry out the exercise-training programme with the patient.Lower limb exercises designed to patient's ability. Emphasis on achieving stability, gait velocity and strength D: 8 weeks F: Training the caregiver on a weekly basisExercises patientcaregiver 35 min daily

I: Intensified Transition Concept:

Psycho-educational seminar for family carers.Individual training course on bedside skills.Therapeutic weekend care, accompanied and monitored by an outpatient care service.Telephone counselling to assess the home situation D: Duration of hospitalization plus 3 months after discharge $\mathrm{F}:$ 1-h psycho-educational seminar3 times 45-60 min individual training course 1 therapeutic weekend 1 telephone counselling after 3 months

\section{I: Graded Repetitive Arm} Supplementary Program with Caregiver Support:A self-

administered upper-limb exercise program, using an exercise booklet and a kit tailored to motor impairment level.Exercises included range of motion, strengthening, and fine motor and goal-directed activities. D: 4 weeks F: Explanation of the program 1 hExercises 60 min a day, 6 days per weekCaregiver involvement $>2$ times/week

\section{I: Voluntary Health Education} Program for Carers:Education on stroke and secondary prevention, proper patient positioning in bed and position changing techniques. D: During hospitalization F: One two-

\section{Outcomes (0)}

Measure point (MP)

O: Patients:Basic functional performance (Kl, FAl)Psychological well-being (CSAL)Caregivers:Psychological well-being (SRCB, CSAL) Others (Discharge location) MP: Admission geriatric rehabilitation, 3, 6 and 9 months

O: Patients:Basic functional performance (NEADL, BI) Psychological well-being (HADS, EQ5D) Caregivers: Psychological well-being (CBS, HADS, EQ-5D)Others (initial stroke admission cost) MP: Measured at: Baseline, 6 and 12 months

O: Patients:Basic functional performance (NEADL, BI, RNLI) Extended functional performance (LLFMA, MAS, BBS, 6MWT)Caregivers: Psychological well-being (CSI) MP: Measured at: Baseline, 8 weeks and 3 months

O: Patients:Basic functiona performance (BI, FIM)Extended functional performance (TUG, ASS, FAT) Caregivers:Psychological wellbeing (BSFC, ZDS, GSL)Others (discharge readmission)

MP: Measured at: After intervention, 4 weeks and 6 months

O: Patients:Extended functional performance (CAAl, MAL, GS) MP: Measured at: Baseline and 4 weeks

O: Patients:Basic functional performance (NEADL, BI, MRS) MP: Measured at: After intervention, 3 and 12 months 
Table 1 Characteristics (Continued)

\begin{tabular}{|c|c|c|c|c|}
\hline $\begin{array}{l}\text { Author } \\
\text { Year } \\
\text { Country }\end{array}$ & $\begin{array}{l}\text { Design (D) } \\
\text { Setting (S) } \\
\text { Sample size (SZ) }\end{array}$ & Target patient population & $\begin{array}{l}\text { Intervention (I) } \\
\text { Duration (D) } \\
\text { Frequency (F) }\end{array}$ & $\begin{array}{l}\text { Outcomes }(0) \\
\text { Measure point }(\mathrm{MP})\end{array}$ \\
\hline & & & hour meeting & \\
\hline $\begin{array}{l}\text { Kalra et al. } \\
\text { Patel et al. } \\
\text { 2004UK }\end{array}$ & $\begin{array}{l}\text { D: RCTblock } \\
\text { randomisation } \\
\text { S: Hospital, home } \\
\text { setting } \\
\text { SZ: } 300 \text { patients } \\
300 \text { caregivers }\end{array}$ & $\begin{array}{l}\text { Patient with strokelndependent in } \\
\text { ADL before strokeMedically } \\
\text { stableExpected to return home }\end{array}$ & $\begin{array}{l}\text { I: Training Caregivers of Stroke } \\
\text { Patients:Instructions on common } \\
\text { stroke related problems, hands-on } \\
\text { training in lifting and handling tech- } \\
\text { niques, facilitation of mobility and } \\
\text { transfers, tailored to the needs of in- } \\
\text { dividual patients. } \\
\text { D: During hospitalizationF: } 3-5 \\
\text { session of } 30-45 \text { min1 follow through } \\
\text { session at home }\end{array}$ & $\begin{array}{l}\text { O: Patients:Basic functional } \\
\text { performance (BI, MRS, FAI) } \\
\text { Psychological well-being (HADS, EQ } \\
\text { VAS) Caregivers:Psychological well- } \\
\text { being (CBS, HADS, EQ VAS)Others } \\
\text { (length of stay, cost, readmission, } \\
\text { mortality, discharge destination) } \\
\text { MP: Measured at: Baseline, 1, 3, } 6 \text { and } \\
12 \text { months }\end{array}$ \\
\hline $\begin{array}{l}\text { van den } \\
\text { Berg et al. } \\
2016 \\
\text { Australia }\end{array}$ & $\begin{array}{l}\text { D: RCTS: Three } \\
\text { hospitals and } \\
\text { home setting } \\
\text { SZ: } 63 \text { patients } 63 \\
\text { caregivers }\end{array}$ & $\begin{array}{l}\text { Patient with stroke Early } \\
\text { rehabilitationMobility problemsNo } \\
\text { cognitive problems No depression }\end{array}$ & $\begin{array}{l}\text { I: Caregiver-Mediated Exercises:A } \\
\text { customized exercise app ( } 37 \\
\text { exercises) on a i-pad was provided to } \\
\text { the patient and carer.Tele-rehabilita- } \\
\text { tion services after discharge and } \\
\text { weekly home visits. } \\
\text { D: } 8 \text { weeks (hospital and home) F: } \geq 5 \\
\text { times per week } 30 \text { minweekly } \\
\text { evaluation session with PT }\end{array}$ & $\begin{array}{l}\text { O: Patients:Basic functional } \\
\text { performance (NEADL, BI, MRS) } \\
\text { Extended functional performance } \\
\text { (SISmob, RMI, LL-FMA, MI, TUG, BBS) } \\
\text { Psychological well-being (HADS)Care- } \\
\text { givers:Psychological well-being (CSI, } \\
\text { HADS) Others (Length of stay, Hos- } \\
\text { pital readmission) } \\
\text { MP: Measured at: Baseline, } 8 \text { weeks } \\
\text { and } 12 \text { weeks }\end{array}$ \\
\hline
\end{tabular}

6MWT Six minute walk test, ASS Ashworth Spasticity Scale, BBS Berg Balance Scale, BI Barthel Index, BSFC Burden Scale for Family Carers, CAAI Chedoke Arm and Hand Activity Inventory, CBS Caregiver Burden Scale, CSI Caregiver Strain Index, CSAL Cantril's Self Anchoring Ladder, CSRI Client Service Receipt Inventory, EQ VAS European Quality of Life Visual Analog Scale, EQ-5D European Quality of Life 5 Descriptive, FAI Frenchay Activities Index, FAT Frenchay Arm Test, FIM Functional Independence Measure, GS Grip strength, GSL Giessen Symptom List, HADS Hospital Anxiety and Depression Scale, KI Katz index, MAL Motor Activity Log, MAS Motor Assessment Scale, MI Motricity index, MRS Modified Rankin Scale, NEADL Nottingham Extended ADL, RMI Rivermead mobility index, RNLI Reintegration to Normal Living Index, SISmob Stroke Impact Scale mobility, SRCB Self Rated Caregiver Burden, TUG Timed up and Go, ZDS Zerssen Depression Scale

speed with the Timed up and Go (TUG) [29, 34] and the six-minute walk test (6MWT) [28] and balance with the Berg Balance Scale (BBS) [28, 34]. Upper limb function was measured with the Chedoke Arm and Hand Activity Inventory (CAAI) [30], the grip strength (GS) [30] and the Frenchay Arm Test (FAT) [29]. General mobility was measured with the Motor Activity Log (MAL) [30], the Motor Assessment Scale (MAS) [28], Stroke Impact Scale mobility (SISmob) [34], Rivermead mobility index (RMI) [34] and the Motricity index (MI) [34]. Furthermore, the Ashworth Spasticity Scale (ASS) [29] was used to measure spasticity. An overview can be found in Table 3.

\section{Psychological well-being}

Caregiver burden was measured in six studies by means of the Caregiver Burden Scale (CBS) [27, 32, 33], the Caregiver Strain Index (CSI) [28, 34], the Self Rated caregiver burden (SRCB) [26], or the Burden Scale for Family Carers (BSFC) [29]. Depression was measured with the Hospital Anxiety and Depression Scale (HADS) in both caregiver and patient [32-34], or with the Zerssen Depression Scale (ZDS) [29]. Furthermore, quality of life was measured with the Euro Quality of Life (EQoL) [27] and the European Quality of Life Visual Analog Scale (EQ VAS) [32, 33]. Other measurements used for psychological well-being were the Giessen Symptom List (GSL) [29] and the Cantril's Self Anchoring Ladder
(CSAL) [26]. An overview can be found in Additional file 3.

\section{Other outcomes}

Healthcare utilization was evaluated as either length of stay [32-34], stroke hospitalization costs [27, 32, 33], total annual health and social care costs [32, 33]. Other outcomes were readmission rate [27, 29, 32-34], mortality [27, 32, 33] and discharge location [26, 27, 32, 33]. An overview can be found in Additional file 4 .

\section{Interventions}

The caregiver interventions ranged from including the caregiver in the decision-making process of discharge planning, to letting the caregiver deliver exercises. All interventions were initiated in the hospital, but their duration ranged from 2 hours to 8 weeks and in three studies the intervention continued after discharge. Studies were grouped into three different types of caregiver intervention.

\section{Care pathway}

One study was categorized in the care pathway group and focused on the process of care. Patients and their caregiver are actively involved in the triage decisions (discharge to a geriatric rehabilitation facility and discharge back to the community) and in the establishment of their care and treatment plan [26]. 
Table 2 Basic functional performance

\begin{tabular}{|c|c|c|c|c|c|c|c|c|c|}
\hline \multirow{2}{*}{$\frac{\text { Study Measure points }}{\text { Everink et al } 2018}$} & & \multicolumn{3}{|l|}{ Basic ADL } & \multicolumn{2}{|l|}{ Extended ADL } & \multicolumn{3}{|l|}{ Others } \\
\hline & & \multicolumn{3}{|l|}{ KI, mean (SD) } & & & \multicolumn{3}{|l|}{ FAI, mean (SD) } \\
\hline $\mathrm{T} 0=$ admission geriatric rehabilitation & & $\mathrm{T} 1$ & \multicolumn{2}{|l|}{$\mathrm{T} 2$} & & & $\mathrm{~T} 1$ & \multicolumn{2}{|l|}{$\mathrm{T} 2$} \\
\hline $\mathrm{T} 1=3$ months & IG & $4.6(2.4)$ & \multicolumn{2}{|l|}{$4.4(2.9)$} & & & $31.1(9.4)$ & \multicolumn{2}{|c|}{$31.0(9.4)$} \\
\hline \multirow[t]{2}{*}{$\mathrm{T} 2=9$ months } & CG & $5.7(2.8)$ & \multicolumn{2}{|l|}{$5.0(3.0)$} & & & $27.4(9.7)$ & \multicolumn{2}{|c|}{$29.4(11.2)$} \\
\hline & & $p=0.360$ & \multicolumn{2}{|l|}{$p=0.862$} & & & $p=0.014$ & \multicolumn{2}{|c|}{$p=0.288$} \\
\hline \multicolumn{5}{|l|}{ Forster et al 2013} & \multicolumn{2}{|c|}{ NEADL, mean (SE) } & & & \\
\hline $\mathrm{T} 0=$ baseline & & & & & \multicolumn{2}{|l|}{$\mathrm{T} 1$} & & & \\
\hline $\mathrm{T} 1=6$ months & IG & & & & \multicolumn{2}{|l|}{$27.4(1.00)$} & & & \\
\hline \multirow[t]{2}{*}{$\mathrm{T} 2=12$ months } & \multirow[t]{2}{*}{ CG } & & & & \multicolumn{2}{|l|}{$27.6(0.99)$} & & & \\
\hline & & & & & \multicolumn{2}{|l|}{$p=0.866$} & & & \\
\hline Galvin et al 2011 & & \multicolumn{3}{|c|}{$\mathbf{B I}$, mean change (SD) } & NEADL, mean & change (SD) & RNLI, mean cha & ange $(S D)$ & \\
\hline $\mathrm{T} 0=$ baseline & & $\mathrm{T} 1-\mathrm{T} 0$ & $\mathrm{~T} 2-\mathrm{T} 1$ & & $\mathrm{~T} 2-\mathrm{T} 1$ & & $\mathrm{~T} 2-\mathrm{T} 1$ & & \\
\hline $\mathrm{T} 1=8$ weeks & IG & $32.3(24)$ & $3.8(8.3)$ & & $7.6(8.3)$ & & $4.7(4.3)$ & & \\
\hline $\mathrm{T} 2=3$ months & CG & $16.3(14.2)$ & $1.5(11.6)$ & & $3.6(7.8)$ & & $0.4(2.9)$ & & \\
\hline & & $p=0.04$ & $p=0.36$ & & $p=0.02$ & & $p=0.00$ & & \\
\hline Gräsel et al 2005 & & BI, mean chang & e $(S D)$ & & & & & & \\
\hline & & $\mathrm{T} 2-\mathrm{T} 0$ & & & & & & & \\
\hline $\mathrm{T} 0=$ after intervention & IG & $11.4(14.1)$ & & & & & & & \\
\hline $\mathrm{T} 2=6$ months & CG & $11.2(16.4)$ & & & & & & & \\
\hline & & $p=0.968$ & & & & & & & \\
\hline & & FIM, mean char & hge (SD) & & & & & & \\
\hline & & $\mathrm{T} 2-\mathrm{T} 0$ & & & & & & & \\
\hline & IG & $2.5(12.9)$ & & & & & & & \\
\hline & CG & $7.4(12.2)$ & & & & & & & \\
\hline & & $p=0.129$ & & & & & & & \\
\hline Hebel et al 2014 & & BI, median & & & NEADL, median & & MRS, median & & \\
\hline $\mathrm{T} 0=$ after intervention & & T0 & $\mathrm{T} 1$ & $\mathrm{~T} 2$ & $\mathrm{~T} 1$ & $\mathrm{~T} 2$ & T0 & $\mathrm{T} 1$ & $\mathrm{~T} 2$ \\
\hline $\mathrm{T} 1=3$ months & $\mathrm{IG}$ & 60 & 75 & 90 & 7 & 14 & 3 & 2 & 2 \\
\hline $\mathrm{T} 2=12$ months & CG & 72.5 & 85 & 90 & 13 & 14.5 & 2 & 2 & 2 \\
\hline & & $p=0.02$ & $p=0.07$ & $p=0.65$ & $p=0.004$ & $p=0.27$ & $p=0.11$ & $p=0.18$ & $p=0.53$ \\
\hline Kalra/Patel et al 2004 & & BI, $\mathrm{BI}>18$ & & & & & FAI, median (IQ & & \\
\hline $\mathrm{T} 0=$ baseline & & $\mathrm{T} 2$ & $\mathrm{~T} 4$ & & & & T0 & $\mathrm{T} 4$ & \\
\hline $\mathrm{T} 2=12$ weeks & $\mid \mathrm{G}$ & $51.3 \%$ & $61.5 \%$ & & & & $25(20-29)$ & $15(9-2$ & \\
\hline $\mathrm{T} 4=52$ weeks & CG & $34.8 \%$ & $50.3 \%$ & & & & $24(21-29)$ & $16(8-2$ & \\
\hline & & $p=0.007$ & $p=0.07$ & & & & $p=$ not stated & $p=$ not & tated \\
\hline van den Berg et al 2016 & & BI, mean $(95 \%$ & & & NEADL, mean & $95 \%$ Cl) & & & \\
\hline $\mathrm{T} 0=$ baseline & & $\mathrm{T} 1$ & $\mathrm{~T} 2$ & & $\mathrm{~T} 1$ & $\mathrm{~T} 2$ & & & \\
\hline $\mathrm{T} 1=8$ weeks & $\mathrm{IG}$ & $89.3(81.6-97)$ & $89.4(81.7$ & $7-97.1)$ & $14.3(12.1-16.4)$ & $15.9(13.8-18.1)$ & & & \\
\hline $\mathrm{T} 2=12$ weeks & CG & $84.9(78.7-91)$ & $88.7(82.4$ & $4-94.9)$ & $10.7(9-12.4)$ & $12.9(11.1-14.6)$ & & & \\
\hline & & $p=0.3811$ & $p=0.889$ & & $p=0.0118$ & $p=0.0319$ & & & \\
\hline
\end{tabular}

IG Intervention Group, CG Control Group, KI Katz Index, BI Barthel Index, FIM Functional Independence Measure, NEADL Nottingham Extended Activities of Daily Living, FAl Frenchay Activity Index, RNL/ Reintegration to Normal Living Index, MRS Modified Rankin Scale 
Table 3 Extended functional performance

\begin{tabular}{|c|c|c|c|c|c|}
\hline \multicolumn{2}{|l|}{$\begin{array}{l}\text { Study Measure } \\
\text { points }\end{array}$} & Lower limb Walking & \multicolumn{2}{|c|}{ Upper limb } & \multirow[t]{2}{*}{ Others } \\
\hline Galvin et al. 2011 & & LL-FMA, mean change (SD) & $\begin{array}{l}\text { MAS, mea } \\
(\mathrm{SD})\end{array}$ & n change & \\
\hline $\mathrm{T} 0=$ baseline & & T1- To & T1-T0 & $\mathrm{T} 2-\mathrm{T} 1$ & T1-T0 \\
\hline $\mathrm{T} 1=8$ weeks & IG & $1.6(2.4)$ & $11.9(7.8)$ & $37.9(9.7)$ & $22.8(18.1)$ \\
\hline \multirow[t]{7}{*}{$\mathrm{T} 2=3$ months } & CG & $1.75(6.3)$ & $4.75(6.2)$ & $\begin{array}{l}35.2 \\
(10.8)\end{array}$ & $9(9)$ \\
\hline & & $p=0.01$ & \multirow{6}{*}{$p=0.00$} & $p=0.59$ & \multirow[t]{6}{*}{$p=0.02$} \\
\hline & & 6MWT, mean change (SD) & & & \\
\hline & & $\mathrm{T} 2-\mathrm{T} 1$ & & & \\
\hline & IG & $164.1(128.7)$ & & & \\
\hline & \multirow[t]{2}{*}{ CG } & $-3.5(32.7)$ & & & \\
\hline & & $p=0.01$ & & & \\
\hline Gräsel et al. 2005 & & TUG, number possible (\%) & \multicolumn{2}{|c|}{$\begin{array}{l}\text { FAT, mean change } \\
\text { (SD) }\end{array}$} & ASS, mean \\
\hline $\mathrm{T} 0=\mathrm{after}$ intervention & & $\mathrm{T} 2$ & \multicolumn{2}{|l|}{ T2-T0 } & T2-T0 \\
\hline $\mathrm{T} 1=4$ weeks & IG & $31(94 \%)$ & \multicolumn{2}{|l|}{$0.3(1.5)$} & $0.3(1.0)$ \\
\hline \multirow[t]{2}{*}{$\mathrm{T} 2=6$ months } & CG & $22(76 \%)$ & \multicolumn{2}{|l|}{$0.2(0.8)$} & $0.0(1.0)$ \\
\hline & & $p=0.960$ & \multicolumn{2}{|l|}{$\begin{array}{l}p= \\
0.679\end{array}$} & $p=0.27$ \\
\hline \multicolumn{2}{|l|}{ Harris et al. 2010} & & \multicolumn{2}{|c|}{$\begin{array}{l}\text { MAL, mean change } \\
\text { (SD) }\end{array}$} & GS, mean $\mathrm{C}$ \\
\hline \multicolumn{2}{|l|}{$\mathrm{T} 0=$ baseline } & & \multicolumn{2}{|l|}{ T1-T0 } & T1-T0 \\
\hline \multirow[t]{7}{*}{$\mathrm{T} 1=4$ weeks } & \multicolumn{2}{|l|}{ IG } & \multicolumn{2}{|l|}{$2.1(0.72)$} & $5.8(3.1)$ \\
\hline & \multicolumn{2}{|l|}{$C G$} & \multicolumn{2}{|l|}{$1.0(0.78)$} & $3.4(2.4)$ \\
\hline & & & \multicolumn{2}{|l|}{$\begin{array}{l}p= \\
0.024\end{array}$} & $p=0.034$ \\
\hline & & & \multicolumn{2}{|c|}{$\begin{array}{l}\text { CAAI, mean change } \\
\text { (SD) }\end{array}$} & \\
\hline & & & \multicolumn{2}{|l|}{ T1-T0 } & \\
\hline & IG & & \multicolumn{2}{|l|}{$20.6(6.1)$} & \\
\hline & CG & & \multicolumn{2}{|l|}{$15.0(7.3)$} & \\
\hline
\end{tabular}

van den Berg et al. 2016

$\mathrm{TO}=$ baseline

$\mathrm{T} 1=8$ weeks

$\mathrm{T} 2=12$ weeks
LL-FMA, mean (95\% Cl)

$\begin{array}{llll} & \text { T0 } & \text { T1 } & \text { T2 } \\ \text { IG } \begin{array}{l}19.4(16.3- \\ \text { 22.6) }\end{array} & 26.1(23-29.2) & 26.1(22.9- \\ \text { CG } & \begin{array}{l}17.2(14.8- \\ 19.7)\end{array} & 22.4(19.3- & 27.6(24.9- \\ & p=0.2654 & p=0.0721 & p=0.3) \\ & p=0.4577\end{array}$

TUG, mean $(95 \% \mathrm{Cl})$

$\begin{array}{llll} & \text { T0 } & \text { T1 } & \text { T2 } \\ \text { IG } & \text { 34.2 (28.6- } & 18.2(12.6- & 17.5(11.9- \\ \text { 39.8) } & 23.8) & 23.2) \\ \text { CG } & \begin{array}{l}\text { 44.2 (39.7- } \\ \text { 48.7) }\end{array} & 17.5(12.9- & 14.1(9.2-19) \\ & p=0.1) & \\ & p=0.0075 & p=0.8503 & p=0.3704\end{array}$

\section{$p=$}

BBS, mean $(95 \% \mathrm{Cl})$

$$
\begin{array}{lll}
\text { T0 } & \text { T1 } & \text { T2 } \\
31.8(26-37.6) & 50.2(44.3-56) & 49.8(43.9- \\
& & 55.6) \\
26.7(22.1- & 44.3(39.7-49) & 46.3(41.6-51) \\
31.3) & & \\
p=0.1752 & p=0.1275 & p=0.3681
\end{array}
$$

SISmob, mean $(95 \% \mathrm{Cl})$

$\begin{array}{lll}\text { T0 } & \text { T1 } & \text { T2 } \\ 44.8(36.8- & 82.3(74.3- & 82.5(74.5- \\ 52.8) & 90.3) & 90.4) \\ 43.1(36.8- & 72.5(66-78.9) & 74.7(68.2- \\ 49.4) & & 81.2) \\ p=0.7342 & p=0.06 & p=0.1382\end{array}$


Table 3 Extended functional performance (Continued)

\begin{tabular}{|c|c|c|c|c|c|}
\hline \multirow{3}{*}{$\begin{array}{l}\text { Study Measure } \\
\text { points }\end{array}$} & \multirow[t]{3}{*}{ Lower limb Walking } & \multirow[t]{3}{*}{ Upper limb } & \multicolumn{3}{|l|}{ Others } \\
\hline & & & \multicolumn{3}{|c|}{ RMI, mean $(95 \% \mathrm{Cl})$} \\
\hline & & & T0 & $\mathrm{T} 1$ & $\mathrm{~T} 2$ \\
\hline & IG & & $7.7(6.3-9.1)$ & $12.6(11.2-14)$ & $12.6(11.2-14)$ \\
\hline & CG & & $6.8(5.6-7.9)$ & $\begin{array}{l}11.6(10.5 \text { - } \\
12.8)\end{array}$ & $12(10.8-13.1)$ \\
\hline & & & $p=0.2937$ & $p=0.284$ & $p=0.5245$ \\
\hline & & & \multicolumn{3}{|c|}{ MI, mean $(95 \% \mathrm{Cl})$} \\
\hline & & & T0 & $\mathrm{T} 1$ & $\mathrm{~T} 2$ \\
\hline & IG & & $\begin{array}{l}66.5(60.1- \\
72.9)\end{array}$ & $\begin{array}{l}78.5(72.1 \text { - } \\
84.9)\end{array}$ & $\begin{array}{l}78.9(72.5- \\
85.3)\end{array}$ \\
\hline & CG & & $\begin{array}{l}62.4(57.4- \\
67.5)\end{array}$ & $\begin{array}{l}74.2(69.1 \text { - } \\
79.3)\end{array}$ & $\begin{array}{l}83.5(78.2- \\
88.8)\end{array}$ \\
\hline & & & $p=0.3291$ & $p=0.3058$ & $p=0.2814$ \\
\hline
\end{tabular}

IG Intervention Group, CG Control Group, LL-FMA Lower Limb Fugl Meyer Assessment, TUG Timed Up and Go test, MAS Motor Assessment Scale, FAT Frenchay Arm Test, MAL Motor activity Log, CAAI Chedoke Arm and Hand Activity Inventory, BBS Berg Balance Scale, $6 M W T 6$ minute walk test, ASS Ashworth Spasticity Scale, GS grip strength, SISmob Stroke Impact Scale mobility part, RMI Rivermead Mobility Index, MI Motricity Index

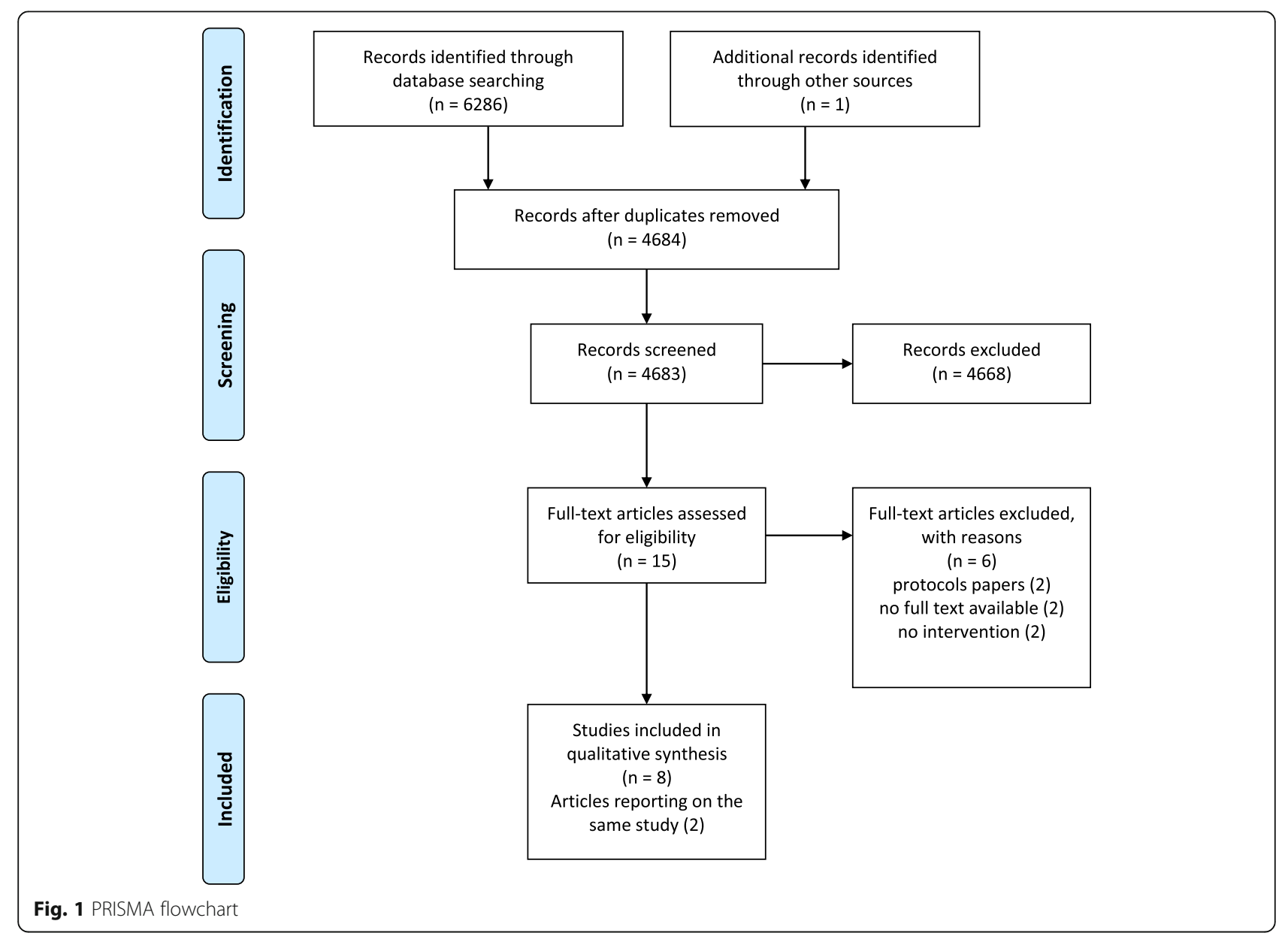




\section{Education and bed-side handling skills}

Four studies evaluated caregiver education and teaching bed-side handling skills, such as education regarding stroke (consequences and prevention) and teaching skills for day-to-day management of stroke survivors like positioning in bed and transfers [27, 29, 31-33]. The duration of these in-hospital interventions was a two-hour meeting [31], 3-5 sessions of 30-45 min [32, 33], or 4 times 45$60 \mathrm{~min}$ [29], Gräsel et al. conducted additional telephone counseling up to 3 months after discharge [29].

\section{Caregiver mediated exercises}

Three studies reported on caregiver mediated exercises, were caregivers assist the patient with a selfadministered exercise program supported with a booklet or an app [28, 30,34]. Galvin et al. studied exercises to strengthen lower limb with the intention of improving balance and gait velocity [28]. Harris et al. focused on improving functionality of the upper limb [30]. Van den Berg et al. studied the exercises aiming to improve gait and gait-related mobility [34]. The duration of these interventions was 4 weeks 60 min a day 6 times per week [30], 8 weeks 35 min a day [28] or 8 weeks $\geq 5$ times per week $30 \mathrm{~min}$ [34]. In two studies the intervention continued after discharge [28, 34].

\section{Impact of intervention types Care pathway}

Everink et al. reported significant improvements for basic functional performance (FAI) and psychological well-being (SRCB) at 3 months, but not at 6 months or 9 months. More intervention patients (83\% versus 58\%, $p=0.004$ ) could be discharged home [26].

\section{Education and bed-side handling skills}

Kalra \& Patel et al. reported significant improvements for basic functional performance at 12 weeks for the BI, but not for the FAI [32, 33]. However, Hebel et al. found significant differences at the end of the hospitalization (approximately 3 days after the intervention in favor of the control group (BI), but these differences faded at follow-up [31]. Gräsel et al. evaluated extended functional performance (TUG, ASS, FAT) but did not observe significant differences [29]. The RCT of Kalra \& Patel et al. found a positive effect on psychological wellbeing for patients and caregivers on all outcome measures used (CBS, HADS, EQVAS) [32, 33], while the RCT of Forster et al. and the non-randomized controlled trial of Gräsel et al. did not (CBS, HADS, EQ-5D, BSFC, GSL, ZDS) [27, 29]. The utilization of healthcare resources was significantly reduced in the study of Kalra \& Patel et al. (length of stay, initial admission costs and total annual health- and social care costs), but not in the study of Forster et al. (stroke hospitalization costs) [27,
$32,33]$. In contrast, more readmissions occurred in the intervention group in the study by Gräsel at 4 weeks, but this difference was not found at 6 months [29].

\section{Caregiver mediated exercises}

Galvin et al. and van den Berg et al. evaluated basic functional performance and found significant improvements in favor of the intervention group, for different outcome measures (BI, NEADL, RNLI) and at different time points $[28,34]$. Significant positive results were found on all outcome measures used for extended functional performance by Galvin et al. (LL-FMA, 6MWT, MAS, BBS) and Harris et al. (MAL, CAAI, grip strength), but not by van den Berg et al. (SIS, RMI, LL-FMA, MI, TUG, BBS) [28, 30, 34]. Psychological well-being was found to be positively influenced in the study of Galvin et al. (CSI). However, van den Berg only found improvement in one of three psychological outcomes (HADS) [28, 34]. Van den Berg et al. also looked at the use of health resources and found significant differences in favor of the intervention group for length of stay and readmission [34].

\section{Discussion}

This review summarizes the evidence of in-hospital and post discharge caregiver involvement in increasing functional performance in older adults. Furthermore, we looked at the influence on psychological well-being, healthcare utilization, discharge destination and mortality. Based on the main caregiver intervention we distinguished three groups, a care pathway [26], education and bed-side handling skills [27, 29, 32, 33], and caregiver mediated exercises [28, 30, 34]. The influence of caregiver involvement on functional performance, as well as on the other outcomes varied widely between the eight included studies. Positive results were mainly evident at the end of the intervention and in the following 3 months.

\section{Care pathway}

The care pathway showed improvements on functional performance of the patients and lowering of the caregiver burden at 3 months. The care pathway might cover the unmet needs of family caregivers that McCusker et al. identified, namely patient medical information, role clarity and support, and reassurance [37]. Good communication about care needs and triage with the caregiver and the patient during hospitalization might make the caregivers more apt to give the support needed. Consequently, this could have positively influenced the number of patients that returned home.

\section{Education and teaching of bed-side handling-skills}

Education on stroke and teaching of bed-side handlingskills showed a positive result on functional performance at 3 months in two of the four studies [32, 33]. Given 
that the other two studies only looked at functional performance at more than 6 months after the intervention, an earlier effect could have been missed [27, 29]. Secondly, best practices for stroke patient and family education, stress the importance of repetition in education [38]. Since training of the bed-side handling skills of the caregiver was only available during hospitalization, there might not have been enough learning opportunities. This was especially the case in de study of Hebel et al. were there was only one contact moment [31]. Also, the process evaluation of the Forster et al. study, showed that in general bed-side and transfer handling-skill were only practiced a few days before discharge and the caregiver mostly observed while therapists were actually performing the transfers [39].

The results on the effect on psychological well-being of education are conflicting. Where Kalra \& Patel et al. found very positive results on all different measurements as well for patients as for caregivers, Forster et al. (using the same intervention) did not [27, 32, 33]. The more personal approach, intervention ownership and intervention fidelity of a small group people who deliver the intervention, compared to a large group of people in a multi-center study might explain these differences [39]. This is in line with Luker et al. who have shown that ownership of an intervention by staff members and patients is important to successfully implement a complex rehabilitation intervention in a clinical trial [40].

Concerning healthcare utilization, the shorter LOS and the lower stroke hospitalization costs in the intervention group of Kalra \& Patel et al. study, could be linked to the better functional performance they found $[32,33]$. This is in line with the studies on older adults in acute care settings where a discharge decision is based on patients functioning [41]. In the study of Forster et al. the discharge date was already known before the intervention was started and thus explains why no shorter LOS was found [39].

\section{Caregiver mediated exercises}

Two of the three studies with caregiver mediated exercise interventions had positive results on all outcomes for functional performance. These results are in line with several reviews on caregiver-mediated exercises for patients with stroke, where extra therapy leads to better functional performance [18, 42, 43]. However, van den Berg only found positive results on two out of eight outcomes namely for the TUG and the NEADL. Looking at the baseline values, the intervention group (IG) of van den Berg et al. performed better compared to the IG of Galvin (an average of 69.6 compared to 56.3 for the BI respectively) $[28,34]$. Patients with lower non-minimal baseline scores have greater potential for improvements [6].
The effect of caregiver mediated exercises on psychological well-being is unclear. These conflicting results might be explained by the many different reasons why a caregiver can experience burden. Besides patient's functional performance there are other factors like anxiety and cognitive function, as well as caregivers' characteristics like an individual's ability to cope with stress, depression, anxiety and physical health [44]. The process evaluation of the study of Galvin et al. showed that the intervention was positively received, it gave structure in daily life and a sense of involvement in the recovery process [45], this explains the positive results they found in favor of the IG for caregiver burden (CSI) [28].

\section{Methodological considerations}

Dividing the studies into groups according to their intervention type results in even smaller numbers of studies. However, we believe this distinction is important as the interventions do differ with regard to content and implementation. The quality of the studies ranges from low till moderate, resulting in low evidence. The different outcome measures used and variation in methodology refrained us from comparing the three different caregiver interventions with each other. Furthermore, seven of the eight studies included were on patients with stroke, and this limits the generalizability of our findings to the geriatric in-hospital and post discharge population.

Despite the small body of evidence regarding caregiver involvement in physiotherapy interventions, the results indicate a potential beneficial effect on several patientrelated outcomes. Nevertheless, more high-quality studies are needed to confirm these findings given the very low grade of evidence, and more attention should be given to process evaluations investigating adherence to the intervention after discharge. Besides questionnaires, objective measurements should be used to evaluate basic functional performance.

\section{Conclusion}

The findings of this review suggest that involvement of caregivers in the rehabilitation of older adults leads to better functional performance up to 3 months after initiation. Three different types of caregiver involvement showed positive results for functional performance up to 3 months after initiation, but evidence is low. Sessions on education and handling skills practice seem effective to improve functional performance and psychological well-being, and decrease length of stay. Caregiver-mediated exercises seem to be more effective when baseline functional performance is lower. However, further research is needed on the generalizability of and contributing factors to, these improvements as programs involving caregivers are so far heterogeneous, predominantly investigated post stroke and show a variable effect on outcomes. 


\section{Supplementary information}

Supplementary information accompanies this paper at https://doi.org/10. 1186/s12877-020-01769-4.

\section{Additional file 1. \\ Additional file 2. \\ Additional file 3 \\ Additional file 4}

\section{Abbreviations}

6MWT: Six minutes' walk test; ASS: Ashworth Spasticity Scale; BBS: Berg Balance Scale; BI: Barthel Index; BSFC: Burden Scale for Family Carers; CAAl: Chedoke Arm and Hand Activity Inventory; CBS: Caregiver Burden Scale; CG: Control Group; CME: Caregiver-Mediated Exercises; CP: Care Pathway; CSI: Caregiver Strain Index; CSAL: Cantril's Self Anchoring Ladder; CSRI: Client Service Receipt Inventory; E\&BHS: Education and Bed-site Handling Skills; EQ: VAS European Quality of Life Visual Analog Scale; EQ5D: European Quality of Life 5 Descriptive; FAl: Frenchay Activities Index; FAT: Frenchay Arm Test; FIM: Functional Independence Measure; GS: Grip strength; GSL: Giessen Symptom List; HADS: Hospital Anxiety and Depression Scale; HCW: Healthcare worker; IG: Intervention group; KI: Katz index; LOS: Length of Stay; MAL: Motor Activity Log; MAS: Motor Assessment Scale; MI: Motricity index; MRS: Modified Rankin Scale; NEADL: Nottingham Extended ADL; PT: Physical therapist; RMI: Rivermead mobility index; RNLI: Reintegration to Normal Living Index; SISmob: Stroke Impact Scale mobility; SRCB: Self Rated Caregiver Burden; TUG: Timed up and Go; ZDS: Zerssen Depression Scale

\section{Acknowledgements}

Mrs. Krizia Tuand. Information specialist Biomedical library, 2Bergen, University Library, KU Leuven, Leuven, Belgium gave support during the development of the search method.

\section{Authors' contributions}

All authors have read and approved the final manuscript. MVD, and JV have taken part in designing and planning of the study, data collection, data analysis and in writing the manuscript. MD has taken part in designing and planning of the study, data analysis and in writing the manuscript. JF, GV and JT have taken part in designing and writing the manuscript. The authors read and approved the final manuscript.

\section{Funding}

No funding was received.

\section{Availability of data and materials \\ Not applicable.}

\section{Ethics approval and consent to participate}

Not applicable.

\section{Consent for publication}

Not applicable.

\section{Competing interests}

The authors declare that they have no competing interests.

\section{Author details}

${ }^{1}$ Department of Physical Medicine and Rehabilitation, UZ Leuven - University Hospitals Leuven, campus Pellenberg, Weligerveld 1, 3212 Pellenberg, Belgium. ${ }^{2}$ Department of Public Health and Primary Care, Geriatrics and Gerontology, KU Leuven, Herestraat 49, 3000 Leuven, Belgium. ${ }^{3}$ Nursing Science (INS), Department of Public Health, University of Basel, Bernoullistrasse 28, 4056 Basel, Switzerland. ${ }^{4}$ Healthcare and Ethics, Faculty of Medicine and Life Sciences, UHasselt, Martelarenlaan 42, 3500 Hasselt, Belgium. ${ }^{5}$ Department of Rehabilitation Sciences, KU Leuven, Tervuursevest 101, 3001 Leuven, Belgium. ${ }^{6}$ Department Geriatric Medicine, UZ Leuven, Herestraat 49, 3000 Leuven, Belgium.
Received: 7 May 2020 Accepted: 10 September 2020

Published online: 22 September 2020

\section{References}

1. Graf C. Functional decline in hospitalized older adults. Am J Nurs. 2006;106(1):58-67.

2. Deschodt M, Wellens NI, Braes T, De Vuyst A, Boonen S, Flamaing J, et al. Prediction of functional decline in older hospitalized patients: a comparative multicenter study of three screening tools. Aging Clin Exp Res. 2011;23(5-6):421-6.

3. Covinsky KE, Palmer RM, Fortinsky RH, Counsell SR, Stewart AL, Kresevic D, et al. Loss of Independence in activities of daily living in older adults hospitalized with medical illnesses: increased vulnerability with age. J Am Geriatr Soc. 2003. https://doi.org/10.1046/j.1532-5415.2003.51152.x.

4. Zisberg A, Shadmi E, Gur-Yaish N, Tonkikh O, Sinoff G. Hospital-associated functional decline: the role of hospitalization processes beyond individual risk factors. J Am Geriatr Soc. 2015. https://doi.org/10.1111/jgs.13193.

5. Sáez De Asteasu ML, Martínez-Velilla N, Zambom-Ferraresi F, Casas-Herrero Á, Lucía A, Galbete A, et al. Physical exercise improves function in acutely hospitalized older patients: secondary analysis of a randomized clinical trial. J Am Med Dir Assoc. 2019;20.7:866-73 Web.

6. Kosse NM, Dutmer AL, Dasenbrock L, Bauer JM, Lamoth CJC. Effectiveness and feasibility of early physical rehabilitation programs for geriatric hospitalized patients: a systematic review. BMC Geriatr. 2013. https://doi.org/ 10.1186/1471-2318-13-107.

7. Hoenig H, Nusbaum N, Brummel-Smith K. Geriatric rehabilitation: state of the art. J Am Geriatr Soc. 2015. https://doi.org/10.1111/j.1532-5415.1997.tb02939.x.

8. Topp R, Fahlman M, Boardley D. Healthy ageing: health promotion and disease prevention. Nurs Clin N Am. 2004. https://doi.org/10.1016/j.cnur.2004.01.007.

9. Smart DA, Dermody G, Coronado ME, Wilson M. Mobility programs for the hospitalized older adult: a scoping review. Gerontol Geriatric Med. 2018. https://doi.org/10.1177/2333721418808146.

10. Gill TM, Gahbauer EA, Han L, Allore HG. Factors associated with recovery of Prehospital function among older persons admitted to a nursing home with disability after an acute hospitalization. J Gerontol. 2009. https://doi. org/10.1093/gerona/glp115.

11. Vochteloo AJH, Moerman S, Tuinebreijer WE, Maier AB, de Vries MR, Bloem RM, et al. More than half of hip fracture patients do not regain mobility in the first postoperative year. Geriatr Gerontol Int. 2012. https://doi.org/10. 1111/j.1447-0594.2012.00904.x.

12. Boyd CM, Landefeld CS, Counsell SR, Palmer RM, Fortinsky RH, Kresevic D, et al. Recovery of activities of daily living in older adults after hospitalization for acute medical illness. J Am Geriatr Soc. 2008. https://doi.org/10.1111/j. 1532-5415.2008.02023.x.

13. Fisher SR, Graham JE, Krishnan S, Ottenbacher KJ. Predictors of 30-day readmission following inpatient rehabilitation for patients at high risk for hospital readmission. Phys Ther. 2016. https://doi.org/10.2522/ptj.20150034.

14. Meesters J, Conijn D, Vermeulen HM, Vliet Vlieland TPM. Physical activity during hospitalization: activities and preferences of adults versus older adults. Physiother Theory Pract. 2019. https:/doi.org/10.1080/09593985.2018.1460429.

15. Holst M, Hansen PL, Pedersen LA, Paulsen S, Valentinsen CD, Køhler M. Physical activity in hospitalized old medical patients; how active are they, and what motivates to physical activity. J Aging Res Clin Practice. 2015;4(2):116-23.

16. Kalapotharakos VI, Diamantopoulos K, Tokmakidis SP. Effects of resistance training and detraining on muscle strength and functional performance of older adults aged 80 to 88 years. Aging Clin Exp Res. 2010. https://doi.org/ 10.1007/BF03324786

17. Sherrington C, Michaleff ZA, Fairhall N, Paul SS, Tiedemann A, Whitney J, et al. Exercise to prevent falls in older adults: an updated systematic review and meta-analysis. Br J Sports Med. 2017:51:1750-8.

18. Peiris $\mathrm{CL}$, Taylor NF, Shield N. Extra physical therapy reduces patient length of stay and improves functional outcomes and quality of life in people with acute or subacute conditions: a systematic review. Arch Phys Med Rehabil. 2011:92(9):1490-500.

19. World Health Organization: Global Health and Aging. US National Institutes of Health Publication 11-7737, 2011. http://www.who.int/ageing/ publications/global_health/en/ Accessed 1 July 2019.

20. Gennuso KP, Gangnon RE, Matthews CE, Thraen-Borowski KM, Colbert LH. Sedentary behavior, physical activity, and markers of health in older adults. Med Sci Sports Exerc. 2013. https://doi.org/10.1249/MSS.0b013e318288a1e5.

21. Seidenfeld SE, Eberle CM, Potter JF. Functional abilities of frail elderly that enable return to the community. Home Health Care Consultant. 2000;7(8):29-32. 
22. Leichsenring K. Developing integrated health and social care services for older persons in Europe. Int J Integr Care. 2004. https://doi.org/10.5334/ijic.107.

23. Sörensen $S$, Pinquart $M$, Duberstein $P$. How effective are interventions with caregivers? An updated meta-analysis. Gerontologist. 2002. https://doi.org/ 10.1093/geront/42.3.356.

24. Sterne JAC, Savović J, Page MJ, Elbers RG, Blencowe NS, Boutron I, et al. RoB 2: a revised tool for assessing risk of bias in randomised trials. BM. 2019;366:4898.

25. Sterne JAC, Hernán MA, Reeves BC, Savović J, Berkman ND, Viswanathan M, et al. ROBINS-l: a tool for assessing risk of bias in non-randomised studies of interventions. BMJ. 2016;355:14919.

26. Everink IHJ, van Haastregt JCM, Tan FES, Schols JMGA, Kempen GIJM. The effectiveness of an integrated care pathway in geriatric rehabilitation among older patients with complex health problems and their informal caregivers: a prospective cohort study. BMC Geriatr. 2018. https://doi.org/10. 1186/s12877-018-0971-4.

27. Forster A, Dickerson J, Young J, Patel A, Kalra L, Nixon J, et al. A structured training Programme for caregivers of inpatients after stroke (TRACS): a cluster randomised controlled trial and cost-effectiveness analysis. Lancet. 2013. https://doi.org/10.1016/s0140-6736(13)61603-7.

28. Galvin R, Cusack T, O'Grady E, Murphy TB, Stokes E. Family-mediated exercise intervention (FAME). Stroke. 2011. https:/doi.org/10.1161/strokeaha.110.594689.

29. Gräsel E, Biehler J, Schmidt R, Schupp W. Intensification of the transition between inpatient neurological rehabilitation and home Care of Stroke Patients. Controlled clinical trial with follow-up assessment six months after discharge. Clin Rehabil. 2005. https://doi.org/10.1191/0269215505cr900oa.

30. Harris JE, Eng JJ, Miller WC, Dawson AS. The role of caregiver involvement in upper-limb treatment in individuals with subacute stroke. Phys Ther. 2010. https://doi.org/10.2522/ptj.20090349.

31. Hebel K, Bieniaszewski L, Kowalewski W. Health education for stroke patient Carers: does it affect functional status improvement in patients after ischemic stroke? Appl Nurs Res. 2014. https://doi.org/10.1016/j.apnr.2014.04.003.

32. Kalra L, Evans A, Perez I, Melbourn A, Patel A, Knapp M, et al. Training carers of stroke patients: randomised controlled trial. BMJ. 2004. https://doi.org/10. 1136/bmj.328.7448.1099.

33. Patel A, Knapp M, Evans A, Perez I, Kalra L. Training care givers of stroke patients: economic evaluation. BMJ. 2004. https://doi.org/10.1136/bmj.328. 7448.1102 .

34. van den Berg M, Crotty M, Liu E, Killington M, Kwakkel G, van Wegen E. Early supported discharge by caregiver-mediated exercises and e-health support after stroke. Stroke. 2016. https://doi.org/10.1161/strokeaha.116.013431.

35. Galvin R, Cusack T, Stokes E. A randomized controlles trial evaluating family mediated exercise (FAME) therapy following stroke. BMC Neurol. 2008. https://doi.org/10.1186/1471-2377-8-22.

36. Forster A, Young J, Nixon J, Karla L, Smithard D, Patel A, et al. A cluster randomized controlled trial of a structured training programme for caregivers of inpatients after stroke (TRACS). Int J Stroke. 2011. https://doi. org/10.1111/j.1747-4949.2011.00722.x.

37. McCusker J, Yaffe M, Lambert SD, Cole M, de Raad M, Belzile E, et al. Unmet needs of family caregivers of hospitalized older adults preparing for discharge home. Chronic IIIn. 2018. https://doi.org/10.1177/ 1742395318789467.

38. Cameron V. Best practices for stroke patient and family education in the acute care setting: a literature review. Medsurg Nurs. 2013;22(1):51-5 64

39. Clarke D, Hwakins R, Sadler E, Harding G, McKevitt C, Godfrey M, et al. Introducing structured caregiver training in stroke care: findings form the TRACS process evaluation study. BMJ Open. 2014. https://doi.org/10.1136/ bmjopen-2013-004473.

40. Luker JA, Craig LE, Bennett L, Ellery F, Langhorne P, Wu O, et al. Implementing a complex rehabilitation intervention in a stroke trial: a qualitative process evaluation of AVERT. BMC Med Res Methodol. 2016. https://doi.org/10.1186/s12874-016-0156-9.

41. Jette DU, Grover L, Keck CP. A qualitative study of clinical decision making in recommending discharge placement from the acute care setting. Phys Ther. 2003:83:224-36

42. Kwakkel G, van Peppen R, Wagenaar RC, Wood Dauphinee S, Richards C, Ashburn A, et al. Effects of augmented exercise therapy time after stroke: a meta-analysis. Stroke. 2004;35(11):2529-39

43. Vloothuis JDM, Mulder M, Veerbeek JM, Konijnenbelt M, Visser-Meily JMA Ket JCF, et al. Caregiver-mediated exercises for improving outcomes after stroke. Cochrane Database Syst Rev. 2016. https://doi.org/10.1002/14651858. CD011058.pub2
44. Zhu W, Jiang Y. A meta-analytic study of predictors for informal caregiver burden in patients with stroke. J Stroke Cerebrovasc Dis. 2018. https://doi. org/10.1016/j.jstrokecerebrovasdis.2018.08.037.

45. Galvin R, Stokes E, Cusack T. Family-mediated exercises (FAME): an exploration of Participant's involvement in a novel form of exercise delivery after stroke. Top Stroke Rehabil. 2014. https://doi.org/10.1310/tsr2101-63.

\section{Publisher's Note}

Springer Nature remains neutral with regard to jurisdictional claims in published maps and institutional affiliations.

\section{Ready to submit your research? Choose BMC and benefit from:}

- fast, convenient online submission

- thorough peer review by experienced researchers in your field

- rapid publication on acceptance

- support for research data, including large and complex data types

- gold Open Access which fosters wider collaboration and increased citations

- maximum visibility for your research: over $100 \mathrm{M}$ website views per year

At BMC, research is always in progress.

Learn more biomedcentral.com/submissions 